\title{
Linearly Convergent Variable Sample-Size Schemes for Stochastic Nash Games: Best-Response Schemes and Distributed Gradient-Response Schemes
}

\author{
Jinlong Lei and Uday V. Shanbhag
}

\begin{abstract}
This paper considers an $N$-player stochastic Nash game in which the $i$ th player minimizes a composite objective $f_{i}(x)+r_{i}\left(x_{i}\right)$, where $f_{i}$ is expectation-valued and $r_{i}$ has an efficient prox-evaluation. In this context, we make the following contributions. (i) Under a strong monotonicity assumption on the concatenated gradient map, we derive (optimal) rate statements and oracle complexity bounds for the proposed variable sample-size proximal stochastic gradient-response (VSPGR) scheme; (ii) We overlay (VS-PGR) with a consensus phase with a view towards developing distributed protocols for aggregative stochastic Nash games. Notably, when the samplesize and the number of consensus steps at each iteration grow at a suitable rate, a linear rate of convergence can be achieved; (iii) Finally, under a suitable contractive property associated with the proximal best-response (BR) map, we design a variable sample-size proximal BR (VS-PBR) scheme, where the proximal BR is computed by solving a sample-average problem. If the batch-size for computing the sample-average is raised at a suitable rate, we show that the resulting iterates converge at a linear rate and derive the oracle complexity.
\end{abstract}

\section{INTRODUCTION}

Noncooperative game theory [1], [2] is a branch of game theory that considers the resolution of conflicts among selfish players, each of which tries to optimize its payoff function, given the rivals' strategies. Nash games represent an important subclass of noncooperative games, originating from the seminal work by [3]. Such models have seen wide applicability in a breadth of engineered systems, such as power grids, communication networks, and sensor networks. In this paper, we consider the Nash equilibrium problem (NEP) with a finite set of $N$ players indexed by $i$ where $i \in \mathcal{N} \triangleq\{1, \cdots, N\}$. For any $i \in \mathcal{N}$, the $i$ th player is characterized by a strategy $x_{i} \in \mathbb{R}^{n_{i}}$ and a payoff function $F_{i}\left(x_{i}, x_{-i}\right)$ dependent on its strategy $x_{i}$ and parametrized by rivals' strategies $x_{-i} \triangleq\left\{x_{j}\right\}_{j \neq i}$. If $n \triangleq \sum_{i=1}^{N} n_{i}$ and $x$ denotes the strategy profile, defined as $x \triangleq\left(x_{1}, \cdots, x_{N}\right) \in$ $\mathbb{R}^{n}$. We consider a stochastic Nash game $\mathcal{P}$ where the objective of player $i$, given rivals' strategies $x_{-i}$, is to solve the following stochastic composite optimization problem:

$$
\min _{x_{i} \in \mathbb{R}^{n_{i}}} F_{i}\left(x_{i}, x_{-i}\right) \triangleq f_{i}\left(x_{i}, x_{-i}\right)+r_{i}\left(x_{i}\right) \quad\left(\mathcal{P}_{i}\left(x_{-i}\right)\right)
$$

where $f_{i}(x) \triangleq \mathbb{E}\left[\psi_{i}(x ; \xi(\omega))\right]$, the random variable $\xi$ : $\Omega \rightarrow \mathbb{R}^{d}$ is defined on the probability space $(\Omega, \mathcal{F}, \mathbb{P})$,

Lei and Shanbhag are with the Department of Industrial and Manufacturing Engineering, Pennsylvania State University, University Park, PA 16802, USA jxl800, udaybagepsu.edu. This research was partially supported by the U.S. National Science Foundation grant CMMI-1538605 and CAREER award CMMI-1246887 (Shanbhag). A extended version with proofs may be found on ArXiv and at http://personal.psu.edu/vvs3/papers/CDC_LS_sub.pdf $\psi_{i}: \mathbb{R}^{n} \times \mathbb{R}^{d} \rightarrow \mathbb{R}$ is a scalar-valued function, and $\mathbb{E}[\cdot]$ denotes the expectation with respect to the probability measure $\mathbb{P}$. We restrict our attention to nonsmooth convex Nash games where $f_{i}\left(x_{i}, x_{-i}\right)$ is assumed to be smooth and convex in $x_{i}$ for any $x_{-i}$ while $r_{i}\left(x_{i}\right)$ is assumed to be convex but a possibly nonsmooth function with an efficient prox-evaluation. A Nash equilibrium (NE) of the stochastic Nash game in which the $i$ th player solves the parametrized problem $\left(\mathcal{P}_{i}\left(x_{-i}\right)\right)$ is a tuple $x^{*}=\left\{x_{i}^{*}\right\}_{i=1}^{N} \in \mathbb{R}^{n}$ such that the following holds for each player $i \in \mathcal{N}$ :

$$
F_{i}\left(x_{i}^{*}, x_{-i}^{*}\right) \leq F_{i}\left(x_{i}, x_{-i}^{*}\right) \quad \forall x_{i} \in \mathbb{R}^{n_{i}} .
$$

In other words, $x^{*}$ is an NE if no player can improve the payoff by unilaterally deviating from the strategy $x_{i}^{*}$.

Our focus is two-fold: (i) Development of variable samplesize stochastic proximal gradient-response (PGR) and proximal best-response (PBR) schemes with optimal (deterministic) rates of convergence; (ii) Extension of PGR schemes to distributed (consensus-based) regimes, allowing for resolving aggregative games with a prescribed communication graph, where linear rates of convergence are achieved by combining increasing number of consensus steps with a growing sample-sizes of sampled gradients.

Prior research. We discuss some relevant research on continuous-strategy Nash games and variance reduction schemes for stochastic optimization.

(i) Deterministic Nash games. Early work considered convex Nash games (where players solve convex programs) where the concatenated gradient map is either strongly monotone [4] or merely monotone maps [5], [6]. While the aforementioned schemes utilized gradient-response techniques, best-response schemes reliant on the contractive nature of the best-response map were examined in [7].

(ii) Stochastic Nash games. Regularized stochastic approximation schemes were presented for monotone stochastic Nash games [8] while extensions have been developed to contend with misspecification [9] and the lack of Lipschitzian properties [10]. More recently, sampled bestresponse schemes have been developed in [11] while rate statements and iteration complexity bounds have been provided for a class of inexact stochastic best-response schemes in [12]-[14]. In fact, we draw inspiration from our work in [14] to develop superior rate statements and extensions to distributed regimes. Finally, a.s. and mean convergence of sequences produced by BR schemes was proven in [13], [15] for stochastic and misspecified potential games.

(iii) Consensus-based distributed schemes for Nash games. Inspired by the advances in consensus-based protocols for re- 
solving distributed optimization problems, Koshal et al. [16] developed two sets of distributed algorithms for monotone aggregative Nash games on graphs. More recently, in [17], [18], the authors combine gradient-based schemes with consensus protocols to address generalized Nash games.

(iv) Variance reduction schemes for stochastic optimization. There has been an effort to utilize increasing batchsizes of sampled gradients in stochastic gradient schemes, leading to improved rates of convergence, as seen in strongly convex [19]-[21] and convex regimes [20]-[23].

\section{Novelty and Contributions.}

(i). VS-PGR. In Section [I, under a strong monotonicity assumption, we prove that a variable sample-size proximal gradient response (VS-PGR) scheme is characterized by a linear rate of convergence in mean-suqared error (Th. 1) while in Th. 2] we establish that the iteration complexity (in terms of proximal evaluations) and oracle complexity to achieve an $\epsilon-\mathrm{NE}$ denoted by $x$ where $x$ satisfies $\mathbb{E}[\| x-$ $\left.x^{*} \|^{2}\right] \leq \epsilon$ are $\mathcal{O}(\ln (1 / \epsilon))$ and $\mathcal{O}\left((1 / \epsilon)^{1+\delta}\right)$, respectively, where $\delta \geq 0$ and $\delta=0$ under a suitable selection of parameters. Furthermore, it is shown in Corollary 1 that with some specific algorithmic parameters, the iteration and oracle complexity to obtain an $\epsilon-\mathrm{NE}$ are bounded by $\mathcal{O}\left(\kappa^{2} \ln (1 / \epsilon)\right)$ and by $\mathcal{O}\left(\kappa^{2} / \epsilon\right)$, where $\kappa$ denotes the condition number.

(ii). Distributed VS-PGR. In Section III addressing an open question in stochastic Nash game, we design a distributed VS-PGR scheme to compute an equilibrium of an aggregative stochastic Nash game over a communication graph. By increasing the number of consensus steps and sample-size at each iteration, this scheme is characterized by a linear rate of convergence (Th. 3). In Th. 4, we show that the iteration, oracle, and communication complexity to compute an $\epsilon$-Nash equilibrium are $\mathcal{O}(\ln (1 / \epsilon)), \mathcal{O}\left((1 / \epsilon)^{1+\delta}\right)$, and $\mathcal{O}\left(\ln ^{2}(1 / \epsilon)\right)$ respectively, where $\delta \geq 0$ and $\epsilon-\mathrm{NE}_{2}$ denotes an $x$ satisfying $\mathbb{E}\left[\left\|x-x^{*}\right\|\right] \leq \epsilon$.

(iii). VS-PBR. In Section IV] we develop a variable sample-size proximal BR (VS-PBR) scheme (see Alg. 2) to solve a class of stochastic Nash games with contractive proximal BR maps, where each player solves a sampleaverage best-response problem per step. We show in Th. 5 that the generated iterates converge to the NE in mean at a linear rate under suitable number of scenarios, and also establish that the iteration and oracle complexity to achieve an $\epsilon-\mathrm{NE}_{2}$ are $\mathcal{O}\left((\ln (1 / \epsilon))\right.$ and $\mathcal{O}\left((1 / \epsilon)^{2(1+\delta)}\right)$ with $\delta \geq 0$.

Notation: When referring to a vector $x$, it is assumed to be a column vector while $x^{T}$ denotes its transpose. Generally, $\|x\|$ denotes the Euclidean vector norm, i.e., $\|x\|=\sqrt{x^{T} x}$. We write a.s. as the abbreviation for "almost surely". For a real number $x$, we define by $\lceil x\rceil$ the smallest integer greater than $x$. For a closed convex function $r(\cdot)$, the proximal operator is defined in the following for any $\alpha>0$ :

$$
\operatorname{prox}_{\alpha r}(x) \triangleq \underset{y}{\operatorname{argmin}}\left(r(y)+\frac{1}{2 \alpha}\|y-x\|^{2}\right) .
$$

For simplicity, $\xi$ denotes $\xi(\omega)$ and in a slight abuse of notation, $N$ denotes the number of players while $N_{k}$ denotes the batch-size of sampled gradients at iteration $k$.

\section{VARIABLE SAMPLE-SIZE GRADIENT RESPONSE}

This section considers the development of a variable sample-size stochastic gradient response scheme for a class of strongly monotone Nash games associated with a strongly monotone concatenated gradient map. We proceed to show that this scheme produces a sequence of iterates that converges to the Nash equilibrium at a linear rate and establish the oracle complexity to achieve an $\epsilon$-Nash equilibrium.

\section{A. Variable sample-size proximal GR (VS-PGR)}

We impose the following assumptions on $\mathcal{P}$.

Assumption 1: Let the following hold.

(a) The function $r_{i}$ is lower semicontinuous and convex with effective domain denoted by $\mathcal{R}_{i} \triangleq \operatorname{dom}\left(r_{i}\right)$. Suppose $\mathcal{R} \triangleq$ $\prod_{j=1}^{N} \mathcal{R}_{i}$ and $\mathcal{R}_{-i}=\prod_{j \neq i} \mathcal{R}_{j}$.

(b) $f_{i}\left(x_{i}, x_{-i}\right)$ is $\mathrm{C}^{1}$ and convex in $x_{i}$ over on an open set containing $\mathcal{R}_{i}$ for every fixed $x_{-i} \in \mathcal{R}_{-i}$;

(c) For all $x_{-i} \in \mathcal{R}_{-i}$ and any $\xi \in \mathbb{R}^{d}, \psi_{i}\left(x_{i}, x_{-i} ; \xi\right)$ is differentiable in $x_{i}$ over an open set containing $\mathcal{R}_{i}$ such that $\nabla_{x_{i}} f_{i}\left(x_{i}, x_{-i}\right)=\mathbb{E}\left[\nabla_{x_{i}} \psi_{i}\left(x_{i}, x_{-i} ; \xi\right)\right]$.

If $G(x ; \xi) \triangleq\left(\nabla_{x_{i}} \psi_{i}(x ; \xi)\right)_{i=1}^{N}$ and $G(x) \triangleq \mathbb{E}[G(x ; \xi)]$, then $G(x)=\left(\nabla_{x_{i}} f_{i}(x)\right)_{i=1}^{N}$ by Assumption 1(iii). The following lemma establishes a tuple $x^{*}$ is an NE of $\mathcal{P}$ if and only if it is a fixed point of a suitable map.

\section{Lemma 1 (Equivalence between $\mathrm{NE}$ and fixed point):}

Given the stochastic Nash game $\mathcal{P}$, suppose Assumption 1 holds for each player $i \in \mathcal{N}$. Define $r(x) \triangleq\left(r_{i}\left(x_{i}\right)\right)_{i=1}^{N}$. Then $x^{*} \in X$ is an NE if and only if $x^{*}$ is a fixed point of $\operatorname{prox}_{\alpha r}(x-\alpha G(x))$, i.e.,

$$
x^{*}=\operatorname{prox}_{\alpha r}\left(x^{*}-\alpha G\left(x^{*}\right)\right), \quad \forall \alpha>0 .
$$

Suppose the iteration index is denoted by $k$ and player $i$ 's strategy at time $k$ is denoted by $x_{i, k} \in \mathbb{R}^{n_{i}}$, which is an estimate of its equilibrium strategy $x_{i}^{*}$. We consider a variable sample-size generalization of the standard proximal stochastic gradient method, in which $N_{k}$ sampled gradients are utilized at iteration $k$. Given a sample $\xi_{k}^{1}, \cdots, \xi_{k}^{N_{k}}$ of $N_{k}$ realizations of the random vector $\xi$, for any $i \in \mathcal{N}$, given $x_{i, 0} \in \mathcal{R}_{i}$, player $i$ updates $x_{i, k+1}$ as follows:

$$
x_{i, k+1}=\operatorname{prox}_{\alpha r_{i}}\left[x_{i, k}-\alpha \frac{\sum_{p=1}^{N_{k}} \nabla_{x_{i}} \psi_{i}\left(x_{k} ; \xi_{k}^{p}\right)}{N_{k}}\right],
$$

where $\alpha>0$ is the constant step size, $\nabla_{x_{i}} \psi_{i}\left(x_{k} ; \xi_{k}^{p}\right), p=$ $1, \cdots, N_{k}$ denote the sampled gradients. Define $w_{k}^{p} \triangleq$ $G\left(x_{k} ; \xi_{k}^{p}\right)-G\left(x_{k}\right)$, and $\bar{w}_{k, N_{k}} \triangleq \frac{1}{N_{k}} \sum_{p=1}^{N_{k}} w_{k}^{p}$. Then the aforementioned scheme can be expressed as

$$
x_{k+1}=\operatorname{prox}_{\alpha r}\left[x_{k}-\alpha\left(G\left(x_{k}\right)+\bar{w}_{k, N_{k}}\right)\right] .
$$

(VS-PGR)

We make the following assumptions on the gradient map and the noise.

Assumption 2: (i) The mapping $G(x)$ is Lipschitz continuous over the set $\mathcal{R}$ with a constant $L$, namely,

$$
\|G(x)-G(y)\| \leq L\|x-y\| \quad \forall x, y \in \mathcal{R} .
$$

(ii) $G(x)$ is strongly monotone with parameter $\eta$, i.e.,

$$
(G(x)-G(y))^{T}(x-y) \geq \eta\|x-y\|^{2} \quad \forall x, y \in \mathcal{R} .
$$


(iii) There exists a constant $\nu$ such that the following holds for any $k \geq 0: \mathbb{E}\left[\bar{w}_{k, N_{k}} \mid \mathcal{F}_{k}\right]=0, \mathbb{E}\left[\left\|\bar{w}_{k, N_{k}}\right\|^{2} \mid \mathcal{F}_{k}\right] \leq$ $\nu^{2} / N_{k} \quad$ a.s., where $\mathcal{F}_{k} \triangleq \sigma\left\{x_{0}, x_{1}, \cdots, x_{k}\right\}$.

\section{B. Rate analysis}

We begin with a simple recursion for the conditional mean squared error in terms of sample size $N_{k}$, step size $\alpha$, and the problem parameters.

Lemma 2: Consider VS-PGR and let Assumptions 1 and 2 hold. Define $q \triangleq 1-2 \alpha \eta+\alpha^{2} L^{2}$. Then for all $k \geq 0$,

$$
\mathbb{E}\left[\left\|x_{k+1}-x^{*}\right\|^{2} \mid \mathcal{F}_{k}\right] \leq q\left\|x_{k}-x^{*}\right\|^{2}+\alpha^{2} \nu^{2} / N_{k}, \quad \text { a.s. }
$$

Using Lemma 2, we are able to show the linear convergence rate of algorithm (VS-PGR).

Theorem 1 (Linear convergence rate of VS-PGR): Let (VS-PGR be applied to $\mathcal{P}$, where $N_{k}=\left\lceil\rho^{-(k+1)}\right\rceil$ for some $\rho \in(0,1)$, and $\mathbb{E}\left[\left\|x_{0}-x^{*}\right\|^{2}\right] \leq C$ for some constant $C>0$. Suppose Assumptions 1 and 2 hold, and $\alpha<2 \eta / L^{2}$. Define $q \triangleq 1-2 \alpha \eta+\alpha^{2} L^{2}$. Then the following holds for any $k \geq 0$.

(i) If $\bar{\rho} \neq q$, then $\mathbb{E}\left[\left\|x_{k}-x^{*}\right\|^{2}\right] \leq C(\rho, q) \max \{\rho, q\}^{k}$, where $C(\rho, q) \triangleq C+\frac{\alpha^{2} \nu^{2}}{1-\min \{\rho / q, q / \rho\}}$.

(ii) If $\rho=q$, then for any $\tilde{\rho} \in(\rho, 1), \mathbb{E}\left[\left\|x_{k}-x^{*}\right\|^{2}\right] \leq \widetilde{D} \tilde{\rho}^{k}$, where $\widetilde{D} \triangleq\left(C+\frac{\alpha^{2} \nu^{2}}{\ln \left((\tilde{\rho} / \rho)^{e}\right)}\right)$.

\section{Iteration and Oracle Complexity}

Next, we examine the iteration (in terms of proximal evaluations) and oracle complexity of this scheme to compute an $\epsilon$-Nash equilibrium, defined next. Recall that a random strategy profile $x: \Omega \rightarrow \mathbb{R}^{n}$ is an $\epsilon-\mathrm{NE}$ if $\mathbb{E}\left[\left\|x-x^{*}\right\|^{2}\right] \leq \epsilon$.

Theorem 2 (Iteration and Oracle Complexity): Let

(VS-PGR be applied to $\mathcal{P}$, where $N_{k}=\left\lceil\rho^{-(k+1)}\right\rceil$ for some $\rho \in(0,1)$, and $\mathbb{E}\left[\left\|x_{0}-x^{*}\right\|^{2}\right] \leq C$. Suppose Assumptions 1 and 2 hold. Define $q \triangleq 1-\underset{\sim}{2} \alpha \eta+\alpha^{2} L^{2}$. Let $\alpha<2 \eta / L^{2}, \tilde{\rho} \in(\rho, 1), C(\rho, q)$ and $\widetilde{D}$ be defined in Theorem 1 Then the number of proximal evaluations needed to obtain an $\epsilon-\mathrm{NE}$ is bounded by $K(\epsilon)$, defined as

$$
K(\epsilon) \triangleq \begin{cases}\frac{1}{\ln (1 / q)} \ln \left(\frac{C(\rho, q)}{\epsilon}\right) & \text { if } \rho<q<1, \\ \frac{1}{\ln (1 / \tilde{\rho})} \ln \left(\frac{\widetilde{D}}{\epsilon}\right) & \text { if } q=\rho, \\ \frac{1}{\ln (1 / \rho)} \ln \left(\frac{C(\rho, q)}{\epsilon}\right) & \text { if } q<\rho<1,\end{cases}
$$

and the number of sampled gradients required is bounded by $M(\epsilon)$, defined as

$$
M(\epsilon) \triangleq \begin{cases}\frac{1}{\rho \ln (1 / \rho)}\left(\frac{C(\rho, q)}{\epsilon}\right)^{\frac{\ln (1 / \rho)}{\ln (1 / q)}}+K(\epsilon) \text { if } \rho<q<1, \\ \frac{1}{\rho \ln (1 / \rho)}\left(\frac{\widetilde{D}}{\epsilon}\right)^{\frac{\ln (1 / \rho)}{\ln (1 / \tilde{\rho})}}+K(\epsilon) & \text { if } q=\rho \\ \frac{1}{\rho \ln (1 / \rho)}\left(\frac{C(\rho, q)}{\epsilon}\right)+K(\epsilon) & \text { if } q<\rho<1 .\end{cases}
$$

Proof. We first consider the case $\rho \neq q$. By Theorem 1(i), the following holds:

$$
\mathbb{E}\left[\left\|x_{k}-x^{*}\right\|^{2}\right] \leq \epsilon \Rightarrow k \geq K_{1}(\epsilon) \triangleq \frac{\ln (C(\rho, q) / \epsilon)}{\ln (1 / \max \{\rho, q\})}
$$

Then we achieve the bound given in equation (3) for cases $\rho<q<1$ and $q<\rho<1$. Note that for $\lambda>1$ and any positive integer $K$, the following holds:

$$
\sum_{k=0}^{K} \lambda^{k} \leq \int_{0}^{K+1} \lambda^{x} d x \leq \frac{\lambda^{K+1}}{\ln (\lambda)}
$$

Then we may obtain the following bound:

$$
\sum_{k=0}^{K_{1}(\epsilon)-1} N_{k} \leq \sum_{k=0}^{K_{1}(\epsilon)-1} \rho^{-(k+1)}+K_{1}(\epsilon) \leq \frac{\rho^{-K_{1}(\epsilon)}}{\rho \ln (1 / \rho)}+K_{1}(\epsilon) .
$$

Note that for any $0<\epsilon, p<1, c_{1}>0$, the following holds:

$$
\begin{aligned}
& \rho^{-\frac{\ln \left(c_{1} / \epsilon\right)}{\ln (1 / p)}}=\left(e^{\ln \left(\rho^{-1}\right)}\right)^{\frac{\ln \left(c_{1} / \epsilon\right)}{\ln (1 / p)}} \\
& =e^{\left.\ln \left(c_{1} / \epsilon\right)\right)^{\frac{\ln (1 / \rho)}{\ln (1 / p)}}}=\left(c_{1} / \epsilon\right)^{\frac{\ln (1 / \rho)}{\ln (1 / p)}} .
\end{aligned}
$$

Thus, the number of sampled gradients required to obtain an $\epsilon-\mathrm{NE}$ is bounded by

$$
\frac{1}{\rho \ln (1 / \rho)}\left(\frac{C(\rho, q)}{\epsilon}\right)^{\frac{\ln (1 / \rho)}{\ln (1 / \max \{\rho, q\})}}+K_{1}(\epsilon) \text {. }
$$

Thus, we achieve the bound given in equation (4) for cases $\rho<q<1$ and $q<\rho<1$. The resultd for the case $\rho=q$ can be similarly proved.

Remark 1: (i) The above theorem establishes that the iteration and oracle complexity to achieve an $\epsilon-\mathrm{NE}$ are $\mathcal{O}(\ln (1 / \epsilon))$ and $\mathcal{O}\left((1 / \epsilon)^{1+\delta}\right)$, where $\delta=0$ when $\rho \in(p, 1)$, $\delta=\frac{\ln (q / \rho)}{\ln (1 / q)}$ when $\rho<q<1$, and $\delta=\frac{\ln (\tilde{\rho} / \rho)}{\ln (1 / \tilde{\rho})}$ when $q=\rho$. (ii) Suppose we use an alternative metric to describe the $\epsilon$-Nash equilibrium: $x: \Omega \rightarrow \mathbb{R}^{n}$ is an $\epsilon-\mathrm{NE}_{2}$ if $\mathbb{E}\left[\left\|x-x^{*}\right\|\right] \leq \epsilon$. Then by Jensen's inequality if follows that an $\epsilon^{2}$-NE is also an $\epsilon-\mathrm{NE}_{2}$, and hence by Theorem 2 we obtain that the iteration and oracle complexity to achieve an $\epsilon-\mathrm{NE}_{2}$ are $\mathcal{O}(\ln (1 / \epsilon))$ and $\mathcal{O}\left((1 / \epsilon)^{2(1+\delta)}\right)$, respectively.

Corollary 1: Let VS-PGR be applied to $\mathcal{P}$, where $\mathbb{E}\left[\left\|x_{0}-x^{*}\right\|^{2}\right] \leq C$ for some constant $C>0$. Suppose Assumptions 1 and 2 hold. Define the condition number $\kappa \triangleq$ $\frac{L}{\eta}$. Set $\alpha=\frac{\eta}{L^{2}}$ and $N_{k}=\left\lceil\rho^{-(k+1)}\right\rceil$ with $\rho=1-\frac{1}{2 \kappa^{2}}$. Then the number of proximal evaluations and samples required to obtain an $\epsilon-\mathrm{NE}$ are bounded by $\mathcal{O}\left(\kappa^{2} \ln (1 / \epsilon)\right)$ and by $\mathcal{O}\left(\kappa^{2} / \epsilon\right)$, respectively.

\section{Distributed VS-PGR for AgGREgative Games}

Next, we consider an aggregative game $\mathcal{P}^{\text {agg }}$, where the $i$ th player solves the following parametrized problem:

$$
\min _{x_{i} \in \mathbb{R}^{n_{i}}} F_{i}^{\mathrm{agg}}\left(x_{i}, x_{-i}\right), \quad\left(\mathcal{P}_{i}^{\mathrm{agg}}\left(x_{-i}\right)\right)
$$

where $F_{i}^{\mathrm{agg}}\left(x_{i}, x_{-i}\right) \triangleq f_{i}\left(x_{i}, x_{i}+\bar{x}_{-i}\right)+r_{i}\left(x_{i}\right), \bar{x} \triangleq$ $\sum_{i=1}^{N} x_{i}$ denotes the aggregate of all players' decisions, $\bar{x}_{-i}=\sum_{j=1, j \neq i}^{N} x_{j}$ denotes the aggregate of all players' decisions except player $i$, and $f_{i}\left(x_{i}, x_{i}+\bar{x}_{-i}\right) \triangleq$ $\mathbb{E}\left[\psi_{i}\left(x_{i}, x_{i}+\bar{x}_{-i} ; \xi\right)\right]$ is expectation valued. We impose the following assumptions on the stochastic aggregative game.

Assumption 3: Let the following hold.

(a) The function $r_{i}$ is lower semicontinuous and convex with 
effective domain denoted by $\mathcal{R}_{i}$ required to be compact.

(b) For any $y \in \overline{\mathcal{R}} \triangleq \sum_{i=1}^{N} \mathcal{R}_{i}, f_{i}\left(x_{i}, y\right)$ is $\mathrm{C}^{1}$ and convex in $x_{i}$ over an open set containing $\mathcal{R}_{i}$.

(c) For all $y \in \overline{\mathcal{R}}$ and any $\xi \in \mathbb{R}^{d}, \psi_{i}\left(x_{i}, y ; \xi\right)$ is differentiable in $x_{i}$ over an open set containing $\mathcal{R}_{i}$ s.t. $\nabla_{x_{i}} f_{i}\left(x_{i}, y\right)=\mathbb{E}\left[\nabla_{x_{i}} \psi_{i}\left(x_{i}, y ; \xi\right)\right]$.

\section{A. Algorithm Design}

We aim to design a distributed algorithm to compute an $\mathrm{NE}$ of $\mathcal{P}^{\text {agg }}$, where each player may exchange information with its local neighbors, and subsequently update its estimate of the equilibrium strategy and the aggregate. The communication among players is defined by an undirected graph $\mathcal{G}=(\mathcal{N}, \mathcal{E})$, where $\mathcal{N} \triangleq\{1, \ldots, N\}$ is the set of players and $\mathcal{E}$ is the set of undirected edges between players. The set of neighbors of player $i$, denoted $\mathcal{N}_{i}$, is defined as $\mathcal{N}_{i} \triangleq\{j \in \mathcal{N}:(i, j) \in \mathcal{E}\}$. Define the adjacency matrix $A=\left[a_{i j}\right]_{i, j=1}^{N}$, where $a_{i j}>0$ if $j \in \mathcal{N}_{i}$ and $a_{i j}=0$, otherwise. A path in $\mathcal{G}$ with length $p$ from $v_{1}$ to $v_{p+1}$ is a sequence of distinct nodes, $v_{1} v_{2} \ldots v_{p+1}$, such that $\left(v_{m}, v_{m+1}\right) \in \mathcal{E}$, for all $m=1, \ldots, p$. The graph $\mathcal{G}$ is termed connected if there is a path between any two distinct players.

Though each player does not have access to all players' decisions, it may estimate the aggregate $\bar{x}$ by communicating with its neighbors. Player $i$ at time $k$ holds an estimate $x_{i, k}$ for its equilibrium strategy and an estimate $v_{i, k}$ for the average of the aggregate. To overcome the fact that the communication network is sparse, we assume that to compute $v_{i, k+1}$, players communicate not once but $\tau_{k}$ rounds at major iteration $k+1$. The strategy of each player is updated by a variable sample-size proximal stochastic gradient scheme that depends on parameters $\alpha$ and $N_{k}$, similar to VS-PGR developed in Section II] We now specify the scheme in Algorithm 1 .

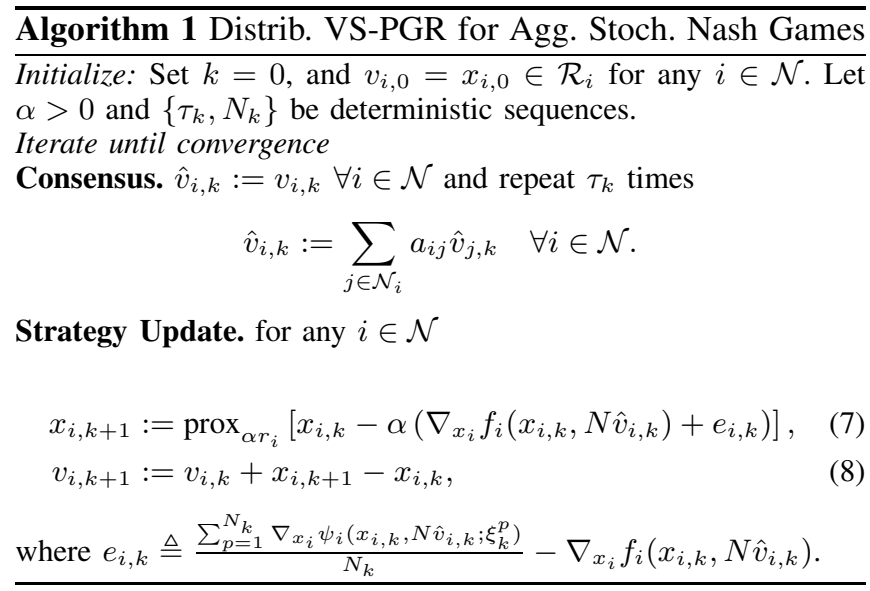

We impose the following conditions on the communication graph, gradient mapping, and observation noise.

Assumption 4: (i) The undirected graph $\mathcal{G}$ is connected and the associated adjacency matrix $A$ is symmetric with row sums equal to one.

(ii) $\phi(x) \triangleq\left(\nabla_{x_{i}} f_{i}\left(x_{i}, \bar{x}\right)\right)_{i=1}^{N}$ is strongly monotone over $\mathcal{R}$ with parameter $\eta$, i.e., $(\phi(x)-\phi(y))^{T}(x-y) \geq \eta \| x-$ $y \|^{2} \quad \forall x, y \in \mathcal{R}$. (iii) The mapping $\phi(x)$ is Lipschitz continuous over $\mathcal{R}$ with a constant $L$, i.e., $\|\phi(x)-\phi(y)\| \leq L\|x-y\| \quad \forall x, y \in \mathcal{R}$. (iv) For any $i \in \mathcal{N}, \nabla_{x_{i}} f_{i}\left(x_{i}, y\right)$ is Lipschitz continuous in $y$ over the set $\overline{\mathcal{R}}$ for every fixed $x_{i} \in \mathcal{R}_{i}$, i.e., there exists some constant $L_{i}$ such that for any $x_{i} \in \mathcal{R}_{i}$,

$\left\|\nabla_{x_{i}} f_{i}\left(x_{i}, y_{1}\right)-\nabla_{x_{i}} f_{i}\left(x_{i}, y_{2}\right)\right\| \leq L_{i}\left\|y_{1}-y_{2}\right\| \quad \forall y_{1}, y_{2} \in \overline{\mathcal{R}}$.

(v) If $\mathcal{F}_{k} \triangleq \sigma\left\{x_{0}, x_{1}, \cdots, x_{k}\right\}$, for any $i \in \mathcal{N}$, there exists a constant $\nu_{i}$ such that the following holds for any $k \geq 0$ :

$$
\mathbb{E}\left[e_{i, k} \mid \mathcal{F}_{k}\right]=0 \text { and } \mathbb{E}\left[\left\|e_{i, k}\right\|^{2} \mid \mathcal{F}_{k}\right] \leq \nu_{i}^{2} / N_{k} \quad \text { a.s. }
$$

\section{B. Convergence Analysis}

Define $A(k) \triangleq A^{\tau_{k}}$. Then by Assumption $4(\mathrm{a}), A(k)$ is also symmetric with the sum of each row equaling one. Note from the consensus step in Algorithm 1 that $\hat{v}_{i, k}=$ $\sum_{j=1}^{N}[A(k)]_{i j} v_{j, k}$. We now recall a prior result.

Lemma 3: By Assumption 4 and [24, Proposition 1], there exists a constant $\theta>0$ and $\beta \in(0,1)$ such that

$$
\left|\left[A^{k}\right]_{i j}-\frac{1}{N}\right| \leq \theta \beta^{k} \quad \forall i, j \in \mathcal{N} .
$$

We introduce the transition matrices $\Phi(k, s)$ from time $s$ to time $k \geq s$ as follows:

$$
\Phi(k, s)=A(k) A(k-1) \cdots A(s), \forall 0 \leq s<k,
$$

where $\Phi(k, k)=A(k)$. We then obtain the following recursion on the mean-squared error.

Proposition 1: Consider Algorithm 1, where $\tau_{k}=k+1$ and $N_{k}=\left\lceil\beta^{-(k+1) / 2}\right\rceil$. Define $M \triangleq \sum_{j=1}^{N} \max _{x_{j} \in \mathcal{R}_{j}}\left\|x_{j}\right\|$, $C_{1} \triangleq M \theta\left(1+2 e \sqrt{1 / \ln \left(\beta^{-1 / 2}\right)}\right), C_{2} \triangleq \frac{4 M \theta}{\ln (1 / \beta)}$, and $\varrho \triangleq$ $\left(1-2 \alpha \eta+2 \alpha^{2} L^{2}\right)$, where $\theta$ and $\beta$ are defined in Lemma 3. Let Assumptions 3 and 4 hold. Then for any $k \geq 0$,

$$
\mathbb{E}\left[\left\|x_{k+1}-x^{*}\right\|^{2}\right] \leq \varrho \mathbb{E}\left[\left\|x_{k}-x^{*}\right\|^{2}\right]+C_{3} \beta^{(k+1) / 2},
$$

where $C_{3}$ is defined as

$$
\begin{aligned}
C_{3} & \triangleq \alpha^{2} \sum_{i=1}^{N} \nu_{i}^{2}+4 \alpha M N\left(C_{1} \beta^{1 / 2}+C_{2}\right) \sum_{i=1}^{N} L_{i} \\
& +4 \alpha^{2} N^{2}\left(C_{1}^{2} \beta^{3 / 2}+C_{2}^{2} \beta^{1 / 2}\right) \sum_{i=1}^{N} L_{i}^{2} .
\end{aligned}
$$

Proof. For purposes of brevity, we merely outline the proof. Firstly, we give a recursion on the conditional mean-squared error as follows:

$$
\begin{aligned}
& \mathbb{E}\left[\left\|x_{k+1}-x^{*}\right\|^{2} \mid \mathcal{F}_{k}\right] \leq \varrho\left\|x_{k}-x^{*}\right\|^{2}+\alpha^{2} \sum_{i=1}^{N} \nu_{i}^{2} / N_{k} \\
& +4 \alpha M N \sum_{i=1}^{N} L_{i}\left\|\hat{v}_{i, k}-y_{k}\right\|+2 \alpha^{2} N^{2} \sum_{i=1}^{N} L_{i}^{2}\left\|\hat{v}_{i, k}-y_{k}\right\|^{2} .
\end{aligned}
$$

We then establish an upper bound on the consensus error:

$$
\left\|y_{k}-\hat{v}_{i, k}\right\| \leq M \theta \beta^{\sum_{p=0}^{k} \tau_{p}}+2 M \theta \sum_{s=1}^{k} \beta^{\sum_{p=s}^{k} \tau_{p}} .
$$

Finally, by getting an upper bound on $\sum_{s=1}^{k} \beta^{\sum_{p=s}^{k} \tau_{p}} \leq$ $e \sqrt{1 / \ln \left(\beta^{-1 / 2}\right)} \beta^{(k+1)(k+2) / 2}+\frac{2 \beta^{(k+1) / 2}}{(k+1) \ln (1 / \beta)}$ and taking the unconditional expectation, we prove the result. 
Based on Prop. 1 we can show the linear rate of convergence of Algorithm 1

Theorem 3 (Linear convergence rate of Algorithm 1): Suppose Assumptions 3 and 4 hold. Consider Algorithm 1 , where $\tau_{k}=k+1, N_{k}=\left[\beta^{-(k+1) / 2}\right]$ and $\mathbb{E}\left[\left\|x_{0}-x^{*}\right\|^{2}\right] \leq C$ for some $C>0$. Let $\alpha \in\left(0, \eta / L^{2}\right)$ and define $\varrho \triangleq\left(1-2 \alpha \eta+2 \alpha^{2} L^{2}\right)$. Then we have the following assertions for any $k \geq 0$.

(i) If $\beta \neq \varrho^{2}$, then $\mathbb{E}\left[\left\|x_{k}-x^{*}\right\|^{2}\right] \leq \widetilde{C}(\varrho, \beta) \max \{\varrho, \sqrt{\beta}\}^{k}$, where $\widetilde{C}(\varrho, \beta) \triangleq C+\frac{C_{3}}{1-\min \{\varrho / \sqrt{\beta}, \sqrt{\beta} / \varrho\}}$ with $C_{3}$ defined in Proposition 1 .

(ii) If $\beta=\varrho^{2}$, then for any $\tilde{\varrho} \in(\varrho, 1), \mathbb{E}\left[\left\|x_{k}-x^{*}\right\|^{2}\right] \leq$ $\widetilde{D}(\varrho) \varrho^{k}$, where $\widetilde{D}(\varrho) \triangleq\left(C+\frac{C_{3}}{\ln \left((\varrho / \varrho)^{e}\right)}\right)$.

Similar to Theorem 2, we may derive bounds on the iteration and oracle complexity as well as the communication complexity to compute an $\epsilon$-Nash equilibrium.

Theorem 4: Suppose Assumptions 3 and 4 hold. Consider Algorithm 1, where $\tau_{k}=k+1, N_{k}=\left\lceil\beta^{-(k+1) / 2}\right\rceil$ and $\mathbb{E}\left[\left\|x_{0}-x^{*}\right\|^{2}\right] \leq C$ for some constant $C>0$. Let $\alpha \in\left(0, \eta / L^{2}\right)$ and define $\varrho \triangleq\left(1-2 \alpha \eta+2 \alpha^{2} L^{2}\right)$. Let $\tilde{\varrho} \in(\varrho, 1), \widetilde{C}(\varrho, \beta)$ and $\widetilde{D}(\varrho)$ be defined in Theorem 3 . Then the number of proximal evaluations needed to obtain an $\epsilon-\mathrm{NE}$ is bounded as follows:

$$
K(\epsilon) \triangleq \begin{cases}\frac{1}{\ln (1 / \varrho)} \ln \left(\frac{\widetilde{C}(\varrho, \beta)}{\epsilon}\right) & \text { if } \beta<\varrho^{2}<1, \\ \frac{1}{\ln (1 / \widetilde{\varrho})} \ln \left(\frac{\widetilde{D}(\varrho)}{\epsilon}\right) & \text { if } \beta=\varrho^{2}, \\ \frac{1}{\ln \left(1 / \beta^{1 / 2}\right)} \ln \left(\frac{\widetilde{C}(\varrho, \beta)}{\epsilon}\right) & \text { if } \varrho<\beta^{1 / 2}<1,\end{cases}
$$

and the round of communications is $\frac{(K(\epsilon)+1)(K(\epsilon)+2)}{2}$, and the number of sampled gradients required is bounded by

$$
M(\epsilon) \triangleq \begin{cases}\frac{\left(\frac{\widetilde{C}(\varrho, \beta)}{\epsilon}\right)^{\frac{\ln \left(1 / \beta^{1 / 2}\right)}{\ln (1 / \varrho)}}}{\beta^{1 / 2} \ln \left(\left(1 / \beta^{1 / 2}\right)\right.}+K(\epsilon) & \text { if } \beta<\varrho^{2}<1, \\ \frac{\left(\frac{\widetilde{D}(\varrho)}{\epsilon}\right)^{\frac{\ln (1 / \varrho)}{\ln (1 / \widetilde{\varrho})}}+K(\epsilon)}{\beta^{1 / 2} \ln \left(\left(1 / \beta^{1 / 2}\right)\right.}+\text { if } \beta=\varrho^{2}, \\ \frac{\left(\frac{\widetilde{C}(\varrho, \beta)}{\epsilon}\right)}{\beta^{1 / 2} \ln \left(\left(1 / \beta^{1 / 2}\right)\right.}+K(\epsilon) & \text { if } \varrho<\beta^{1 / 2}<1 .\end{cases}
$$

Remark 2: Recall that in [25], a fast distributed gradient algorithm based on Nesterov's accelerated gradient algorithm is employed to solve a distributed convex optimization problem, where at each step, $\mathcal{O}(\ln (k))$ consensus steps are taken. In [25], the authors show that in merely convex settings, the rate is $\mathcal{O}\left(1 / k^{2}\right)$ (optimal) and total number of communications rounds is $\mathcal{O}(k \ln (k))$ up to time $k$. Our scheme (Algorithm 1) requires $\mathcal{O}\left(k^{2}\right)$ rounds of communications to recover the optimal linear rate of convergence but does so in a stochastic game-theoretic regime. In future work, we intend to investigate how the number of consensus steps may be chosen to maintain geometric convergence while reducing communication overhead.

\section{VARiable Sample-Size Prox. Best Response}

In this section, we consider the class of stochastic Nash games in which the proximal BR map is contractive [26]. We propose a variable sample size proximal BR scheme for computing an equilibrium, and derive rate statements and establish iteration and oracle complexity bounds.

\section{A. Background on proximal best-reponse maps}

For any $i \in \mathcal{N}$ and any tuple $y \in \mathbb{R}^{n}$, define the proximal BR map $\widehat{x}_{i}(y)$ as $\widehat{x}_{i}(y) \triangleq$ $\operatorname{argmin}_{x_{i} \in \mathbb{R}^{n_{i}}}\left[\mathbb{E}\left[\psi_{i}\left(x_{i}, y_{-i} ; \xi\right)\right]+r_{i}\left(x_{i}\right)+\frac{\mu}{2}\left\|x_{i}-y_{i}\right\|^{2}\right]$.

We impose the following assumption on problem $\left[\mathcal{P}_{i}\left(x_{-i}\right)\right]$.

Assumption 5: (i) Assumption 3/i).

(ii) For every fixed $x_{-i} \in \mathcal{R}_{-i}, f_{i}\left(x_{i}, x_{-i}\right)$ is $\mathrm{C}^{2}$ and convex in $x_{i}$ over on an open set containing $\mathcal{R}_{i}$. Moreover, $\nabla_{x_{i}} f_{i}\left(x_{i}, x_{-i}\right)$ is assumed to be Lipschitz continuous in $x_{i}$ uniformly in $x_{-i}$ with constant $L_{i}$, i.e., for any $x_{i}, x_{i}^{\prime} \in \mathcal{R}_{i}$,

$$
\left\|\nabla_{x_{i}} f_{i}\left(x_{i}, x_{-i}\right)-\nabla_{x_{i}} f_{i}\left(x_{i}^{\prime}, x_{-i}\right)\right\| \leq L_{i}\left\|x_{i}-x_{i}^{\prime}\right\| .
$$

(iii) For all $x_{-i} \in \mathcal{R}_{-i}$ and any $\xi \in \mathbb{R}^{d}, \psi_{i}\left(x_{i}, x_{-i} ; \xi\right)$ is differentiable in $x_{i}$ over an open set containing $\mathcal{R}_{i}$. Moreover, for any $i \in \mathcal{N}$ and all $x \in \mathcal{R}$, there exists $M_{i}>0$ such that $\mathbb{E}\left[\left\|\nabla_{x_{i}} f_{i}(x)-\nabla_{x_{i}} \psi_{i}\left(x_{i}, x_{-i} ; \xi\right)\right\|^{2}\right] \leq M_{i}^{2}$.

By Assumption [5, the second derivatives of the functions $f_{i}, \forall i \in \mathcal{N}$ on $\mathcal{R}$ are bounded. Analogous to the avenue adopted in [26], we may define

$$
\Gamma \triangleq\left(\begin{array}{cccc}
\frac{\mu}{\mu+\zeta_{1, \min }} & \frac{\zeta_{12, \max }}{\mu+\zeta_{1, \min }} & \cdots & \frac{\zeta_{1 N, \max }}{\mu+\zeta_{1, \min }} \\
\frac{\zeta_{21, \max }}{\mu+\zeta_{2, \min }} & \frac{\mu}{\mu+\zeta_{2, \min }} & \cdots & \frac{\zeta_{2 N, \max }}{\mu+\zeta_{2, \min }} \\
\vdots & & \ddots & \\
\frac{\zeta_{N 1, \max }}{\mu+\zeta_{N, \min }} & \frac{\zeta_{N 2, \max }}{\mu+\zeta_{N, \min }} & \cdots & \frac{\mu}{\mu+\zeta_{N, \min }}
\end{array}\right)
$$

where $\zeta_{i, \text { min }} \triangleq \inf _{x \in X} \lambda_{\min }\left(\nabla_{x_{i}}^{2} f_{i}(x)\right)$ and $\zeta_{i j, \max } \triangleq$ $\sup _{x \in X}\left\|\nabla_{x_{i} x_{j}}^{2} f_{i}(x)\right\| \forall j \neq i$. Then by [11, Theorem 4] we obtain that

$$
\left(\begin{array}{c}
\left\|\widehat{x}_{1}\left(y^{\prime}\right)-\widehat{x}_{1}(y)\right\| \\
\vdots \\
\left\|\widehat{x}_{N}\left(y^{\prime}\right)-\widehat{x}_{N}(y)\right\|
\end{array}\right) \leq \Gamma\left(\begin{array}{c}
\left\|y_{1}^{\prime}-y_{1}\right\| \\
\vdots \\
\left\|y_{N}^{\prime}-y_{N}\right\|
\end{array}\right) .
$$

If the spectral radius $\rho(\Gamma)<1$, then the proximal bestresponse map is contractive w.r.t. some monotonic norm. These sufficient conditions for the contractive property of the BR map $\widehat{x}(\bullet)$ can be found in [11], [26].

\section{B. Variable sample-size proximal BR scheme}

Suppose at iteration $k$, we have $N_{k}$ samples $\xi_{k}^{1}, \cdots, \xi_{k}^{N_{k}}$ of the random vector $\xi$. For any $x_{i} \in X_{i}$, we approximate $f_{i}\left(x_{i}, y_{-i, k}\right)$ by $\frac{1}{N_{k}} \sum_{p=1}^{N_{k}} \psi_{i}\left(x_{i}, y_{-i, k} ; \xi_{k}^{p}\right)$ and solve the sample-average best-response problem (14). We then get the variable-size proximal BR scheme (Algorithm 2).

\section{Algorithm 2 Variable-size proximal BR scheme}

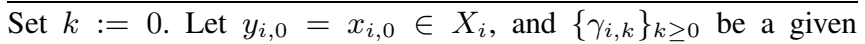
deterministic sequence for $i=1, \ldots, N$.

(1) For $i=1, \ldots, N$, player $i$ updates estimate $x_{i, k+1}$ as

$$
\begin{aligned}
x_{i, k+1}=\underset{x_{i} \in \mathbb{R}^{n_{i}}}{\operatorname{argmin}} & {\left[\frac{1}{N_{k}} \sum_{p=1}^{N_{k}} \psi_{i}\left(x_{i}, y_{-i, k} ; \xi_{k}^{p}\right)\right.} \\
+ & \left.r_{i}\left(x_{i}\right)+\frac{\mu}{2}\left\|x_{i}-y_{i, k}\right\|^{2}\right] .
\end{aligned}
$$

(2) For $i=1, \ldots, N, y_{i, k+1}:=x_{i, k+1}$;

(3) Set $k:=k+1$ and return to (1). 


\section{Oracle and iteration complexity}

Define $\varepsilon_{i, k+1} \triangleq x_{i, k+1}-\widehat{x}_{i}\left(y_{k}\right)$. We may obtain an bound on $\mathbb{E}\left[\left\|\varepsilon_{i, k+1}\right\|^{2}\right]$ in the following lemma.

Lemma 4: Suppose Assumption 5 holds. Consider Algorithm 2. Then $\mathbb{E}\left[\left\|\varepsilon_{i, k+1}\right\|^{2}\right] \leq \frac{M_{i}^{2} C_{r}^{2}}{N_{k}}$, where $C_{r} \triangleq$ $\frac{\mu}{\mu^{2}+L^{2}}\left(1-L / \sqrt{\mu^{2}+L^{2}}\right)^{-1}$ with $L \triangleq \max _{i} L_{i}$.

Proof. Define $\bar{w}_{i, k}\left(x_{i}\right) \triangleq \frac{1}{N_{k}} \sum_{p=1}^{N_{k}} \nabla_{x_{i}} \psi_{i}\left(x_{i}, y_{-i, k} ; \xi_{k}^{p}\right)-$ $\nabla_{x_{i}} f_{i}\left(x_{i}, y_{-i, k}\right)$. By the optimality condition, $x_{i, k+1}$ and $\widehat{x}_{i}\left(y_{k}\right)$ are respectively a fixed point of the map prox $\operatorname{pr}_{i}\left[x_{i}-\right.$ $\left.\alpha\left(\nabla_{x_{i}} \tilde{f}_{i}\left(x_{i}, y_{k}\right)+\bar{w}_{i, k}\left(x_{i}\right)\right)\right]$ and $\operatorname{prox}_{\alpha r_{i}}\left[x_{i}-\alpha \tilde{f}_{i}\left(x_{i}, y_{k}\right)\right]$ for any $\alpha>0$. Then by the nonexpansive property of the proximal operator, the following holds for any $\alpha>0$ :

$$
\begin{aligned}
& \left\|\varepsilon_{i, k+1}\right\| \leq \alpha\left\|\bar{w}_{i, k}\left(x_{i, k+1}\right)\right\| \\
& +\left\|x_{i, k+1}-\widehat{x}_{i}\left(y_{k}\right)-\alpha\left(\tilde{f}_{i}\left(x_{i, k+1}, y_{k}\right)-\tilde{f}_{i}\left(\widehat{x}_{i}\left(y_{k}\right), y_{k}\right)\right)\right\| \\
& \leq \sqrt{(1-\alpha \mu)^{2}+\alpha^{2} L^{2}}\left\|\varepsilon_{i, k+1}\right\|+\alpha\left\|\bar{w}_{i, k}\left(x_{i, k+1}\right)\right\| .
\end{aligned}
$$

In the above inequality, by setting $\alpha=\frac{\mu}{\mu^{2}+L^{2}}$, we obtain that $\left\|\varepsilon_{i, k+1}\right\| \leq C_{r}\left\|\bar{w}_{i, k}\left(x_{i, k+1}\right)\right\|$. Then by using Assumption 5 (iii), the lemma is proved.

Similar to [14, Prp. 4], we can prove the linear rate of convergence. We then establish the iteration and oracle complexity to obtain an $\epsilon-\mathrm{NE}_{2}$, which is random strategy profile $x: \Omega \rightarrow \mathbb{R}^{n}$ satisfies $\mathbb{E}\left[\left\|x-x^{*}\right\|\right] \leq \epsilon$.

Theorem 5: Suppose Ass. 5 holds and $a \triangleq\|\Gamma\|<$ 1. Let Algorithm 2 be applied to the stochastic Nash game $\left(\mathcal{P}_{i}\left(x_{-i}\right)\right)$, where $\mathbb{E}\left[\left\|x_{i, 0}-x_{i}^{*}\right\|\right] \leq C$ and $N_{k}=$ $\left[\frac{\max _{i} M_{i}^{2} C_{r}^{2}}{\eta^{2 k}}\right]$ for some $\eta \in(0,1)$. Define $c \triangleq \max \{a, \eta\}$, let $\tilde{\eta} \in(c, 1)$, and $D=1 / \ln \left((\tilde{\eta} / c)^{e}\right)$. Then the number of the deterministic optimization problems solved and samples required by player $i$ to obtain an $\epsilon-\mathrm{NE}_{2}$ are $\mathcal{O}(\ln (\sqrt{N} / \epsilon))$ and $\mathcal{O}\left((\sqrt{N} / \epsilon)^{\frac{2 \ln (1 / \eta)}{\ln (1 / \tilde{\eta})}}\right)$, respectively.

\section{COnCluding Remarks}

We consider a class of stochastic Nash games where each player-specific objective is a sum of an expectation-valued smooth function and a convex nonsmooth function. We consider three schemes: (i) Variable sample-size proximal gradient response (VS-PGR) for strongly monotone stochastic Nash games; (ii) Distributed VS-PGR for strongly monotone aggregative Nash games; and (iii) VS proximal best-response (VS-PBR) for stochastic Nash games with contractive best response maps. Under suitable assumptions, we show that all schemes generate sequences that converge at the (optimal) linear rate and derive bounds on the computational, oracle, and communication complexity.

\section{REFERENCES}

[1] D. Fudenberg and J. Tirole, Game Theory. MIT Press, 1991.

[2] T. Basar and G. J. Olsder, Dynamic noncooperative game theory. SIAM, 1999, vol. 23.

[3] J. F. Nash, Jr., "Equilibrium points in $n$-person games," Proc. Nat. Acad. Sci. U. S. A., vol. 36, pp. 48-49, 1950.

[4] T. Alpcan and T. Basar, "A game-theoretic framework for congestion control in general topology networks," in Decision and Control, 2002, Proceedings of the 41st IEEE Conference on, vol. 2. IEEE, 2002, pp. 1218-1224.
[5] H. Yin, U. V. Shanbhag, and P. G. Mehta, "Nash equilibrium problems with scaled congestion costs and shared constraints," IEEE Transactions on Automatic Control, vol. 56, no. 7, pp. 1702-1708, 2011.

[6] A. Kannan and U. V. Shanbhag, "Distributed computation of equilibria in monotone Nash games via iterative regularization techniques," SIAM Journal on Optimization, vol. 22, no. 4, pp. 1177-1205, 2012.

[7] F. Facchinei and J. Pang, Nash Equilibria: The Variational Approach. Convex Optimization in Signal Processing and Communication, Cambridge University Press, 2009.

[8] J. Koshal, A. Nedic, and U. V. Shanbhag, "Regularized iterative stochastic approximation methods for stochastic variational inequality problems," IEEE Trans. Automat. Contr., vol. 58, no. 3, pp. 594-609, 2013.

[9] H. Jiang, U. V. Shanbhag, and S. P. Meyn, "Distributed computation of equilibria in misspecified convex stochastic Nash games," IEEE Trans. Automat. Contr., vol. 63, no. 2, pp. 360-371, 2018.

[10] F. Yousefian, A. Nedić, and U. V. Shanbhag, "Self-tuned stochastic approximation schemes for non-Lipschitzian stochastic multi-user optimization and Nash games," IEEE Transactions on Automatic Control, vol. 61, no. 7, pp. 1753-1766, 2016.

[11] J.-S. Pang, S. Sen, and U. V. Shanbhag, "Two-stage non-cooperative games with risk-averse players," Mathematical Programming, vol. 165, no. 1, pp. 235-290, 2017.

[12] U. V. Shanbhag, J.-S. Pang, and S. Sen, "Inexact best-response schemes for stochastic Nash games: Linear convergence and iteration complexity analysis," in Decision and Control (CDC), 2016 IEEE 55th Conference on. IEEE, 2016, pp. 3591-3596.

[13] J. Lei and U. V. Shanbhag, "A randomized inexact proximal bestresponse scheme for potential stochastic nash games," in Decision and Control (CDC), 2017 IEEE 56th Annual Conference on. IEEE, 2017, pp. 1646-1651.

[14] J. Lei, U. V. Shanbhag, J.-S. Pang, and S. Sen, "On synchronous, asynchronous, and randomized best-response schemes for computing equilibria in stochastic Nash games," to appear in Mathematics of Operations Research, 2018.

[15] J. Lei and U. V. Shanbhag, "Asynchronous schemes for stochastic and misspecified potential games and nonconvex optimization," arXiv:1711.03963, 2017.

[16] J. Koshal, A. Nedić, and U. V. Shanbhag, "Distributed algorithms for aggregative games on graphs," Operations Research, vol. 64, no. 3, pp. 680-704, 2016.

[17] P. Yi and L. Pavel, "A distributed primal-dual algorithm for computation of generalized Nash equilibria with shared affine coupling constraints via operator splitting methods," arXiv preprint arXiv:1703.05388. 2017.

[18] G. Belgioioso and S. Grammatico, "Semi-decentralized nash equilibrium seeking in aggregative games with separable coupling constraints and non-differentiable cost functions," IEEE control systems letters, vol. 1, no. 2, pp. 400-405, 2017.

[19] U. V. Shanbhag and J. Blanchet, "Budget constrained stochastic approximation," Proceedings of the Winter Simulation Conference, 2015.

[20] A. Jofré and P. Thompson, "On variance reduction for stochastic smooth convex optimization with multiplicative noise," arXiv preprint arXiv:1705.02969. 2017.

[21] A. Jalilzadeh, U. V. Shanbhag, J. Blanchet, and P. W. Glynn, "Optimal smoothed variable sample-size accelerated proximal methods for structured nonsmooth stochastic convex programs," arXiv preprint arXiv:1803.00718,1, 2018.

[22] A. Jalilzadeh and U. V. Shanbhag, "eg-VSSA: An extragradient variable sample-size stochastic approximation scheme: Error analysis and complexity trade-offs," in Winter Simulation Conference, WSC 2016, 2016, pp. 690-701.

[23] S. Ghadimi and G. Lan, "Accelerated gradient methods for nonconvex nonlinear and stochastic programming," Mathematical Programming, vol. 156, no. 1-2, pp. 59-99, 2016.

[24] A. Nedic and A. Ozdaglar, "Distributed subgradient methods for multiagent optimization," IEEE Transactions on Automatic Control, vol. 54, no. 1, pp. 48-61, 2009.

[25] D. Jakovetić, J. Xavier, and J. M. Moura, "Fast distributed gradient methods," IEEE Transactions on Automatic Control, vol. 59, no. 5, pp. 1131-1146, 2014.

[26] F. Facchinei and J. Pang, Nash equilibria: The variational approach, ser. Convex Optimization in Signal Processing and Communications. Cambridge University Press (Cambridge, England), 2009. 